\title{
INTEGRAL observation of the accreting pulsar GX $1+4^{\star}$
}

\author{
C. Ferrigno ${ }^{1}$, A. Segreto ${ }^{1}$, A. Santangelo ${ }^{2}$, J. Wilms ${ }^{3,6}$, I. Kreykenbohm ${ }^{2,4}$, M. Denis ${ }^{5}$, and R. Staubert ${ }^{2}$ \\ 1 IASF - INAF, via Ugo la Malfa 153, 90136 Palermo, Italy \\ e-mail: ferrigno@ifc.inaf.it \\ 2 IAAT, Abt. Astronomie, Universität Tübingen, Sand 1, 72076 Tübingen, Germany \\ 3 Department of Physics, University of Warwick, Coventry CV4 7AL, UK \\ ${ }^{4}$ INTEGRAL Science Data Centre, 16 Ch. d'Écogia, 1290 Versoix, Switzerland \\ 5 Space Research Center, Bartycka 18a, 00716 Warsaw, Poland \\ ${ }^{6}$ Dr. Remeis Sternwarte, Astronomisches Institut University of Erlangen-Nuremberg, Sternwartstr. 7, 96049 Bamberg, Germany
}

Received 21 July 2005 / Accepted 20 September 2006

\section{ABSTRACT}

\begin{abstract}
Aims. We present the results of the INTEGRAL monitoring campaign on the accreting low mass X-ray binary pulsar GX $1+4$ performed during the Galactic plane scan of the INTEGRAL Core Programme.

Methods. The source was observed in different luminosity states ranging from $L_{20-40 \mathrm{keV}}=1.7 \times 10^{-10} \mathrm{erg} \mathrm{cm}^{-2} \mathrm{~s}^{-1}$, to $L_{20-40 \mathrm{keV}}=$ $10.5 \times 10^{-10} \mathrm{erg} \mathrm{cm}^{-2} \mathrm{~s}^{-1}$ for about $779 \mathrm{ks}$ from March 2003 until October 2004.

Results. Our observations confirm the secular spin down of GX 1+4 with the spin period $\left(P_{\mathrm{s}}\right)$ varying from $139.63 \mathrm{~s}$ to $141.56 \mathrm{~s}$. In the highest luminosity state, a spin-up phase is observed. The phase-averaged spectrum of the source was modelled either with an absorbed cut-off power law or with a Comptonization model with significantly different parameters in the two brightest luminosity states. No evidence of any absorption-like feature is observed in the phase averaged spectrum up to $110 \mathrm{keV}$. At highest luminosity, the source is found to pulsate up to $130 \mathrm{keV}$. Phase resolved spectroscopy reveals a phase-dependent continuum and marginal evidence for an absorption feature at $34 \pm 2 \mathrm{keV}$ in the descending part of the pulse. If interpreted as due to electron resonant cyclotron scattering, the magnetic field in the emitting region would be $(2.9 \pm 0.2) \times 10^{12}(1+z) \mathrm{G}$ where $z$ is the gravitational red shift of the emitting region. We also observed a very low luminosity state, typical of this source, which lasted for about two days during which the source spectrum was modelled by a simple power law, and a pulsed signal was still detectable in the $15-100 \mathrm{keV}$ energy range.
\end{abstract}

Key words. X-rays: stars - stars: pulsars: individual: GX 1+4

\section{Introduction}

The X-ray binary pulsars (XRBPs) were discovered more than 30 years ago with the pioneer observation of Cen X-3 by Giacconi et al. (1971). Even though the basic mechanisms of such a pulsed emission were understood quickly (Pringle \& Rees 1972; Davidson \& Ostriker 1973), XRBPs still present many puzzling aspects. In particular, their wide-band spectral behaviour has not been explained yet on the basis of a unique coherent physical model. Radiation from XRBPs originates from the accretion of ionized gas, from a nearby companion, an $\mathrm{O}$ or B star $\left(M \geq 5 M_{\odot}\right)$ in High Mass X-ray Binaries (HMXRBs), a later than type A star $\left(M \leq M_{\odot}\right)$ or a white dwarf in low mass $\mathrm{X}$-ray binaries (LMXRBs), onto the magnetic pole regions of strongly magnetized $\left(B \sim 10^{12} \mathrm{G}\right)$ rotating neutron stars. At several hundred neutron star radii, the plasma is threaded and then funneled along the magnetic field lines onto the magnetic poles at the neutron star surface (Pringle \& Rees 1972; Davidson \& Ostriker 1973). Pulsation is generated because of the nonalignment between the magnetic and rotational axes. Spin periods are not constant and spin-up and spin-down behaviours are observed on a variety of time scales (Bildsten et al. 1997).

According to accretion theory (Ghosh et al. 1977; Ghosh \& Lamb 1979a,b), the accretion process onto a neutron star surrounded by a disk strongly depends on the structure of the

* Appendices are only available in electronic form at http: //www . aanda.org transition zone where the Keplerian disk meets the pulsar magnetosphere. The occurrence of accretion is mainly governed by the ratio between the magnetospheric radius $r_{\mathrm{m}}$, where the ram pressure of a spherically symmetric inflow equals the magnetic pressure, and the corotation radius $r_{\mathrm{co}}$, where the magnetic field lines move at the local Keplerian velocity.

When $r_{\mathrm{m}}<r_{\mathrm{co}}$, the plasma is forced to stream along the field lines and eventually falls onto the neutron star's magnetic poles. When $r_{\mathrm{m}}>r_{\mathrm{co}}$, the accretion stops due to the onset of the centrifugal barrier, which is known as the propeller effect (Illarionov \& Sunyaev 1975; Stella et al. 1986). If $r_{\mathrm{m}} \sim r_{\mathrm{co}}$, the system is in a near equilibrium state. In this condition, spin-up and spindown periods, due to the transfer of angular momentum from the accreted plasma to the NS, can occur when variations in the accretion rate $(\dot{M})$ take place. When $\dot{M}$ is sufficiently high, the star experiences a spin-up torque; as $\dot{M}$ decreases, the spin-up torque falls and vanishes at a critical accretion rate. For a lower accretion rate, the star experiences a spin-down torque whose magnitude increases until $\dot{M}$ reaches the minimum accretion rate consistent with steady accretion (Ghosh \& Lamb 1979b).

Such torque reversal episodes characterise the peculiar spin history of the $(P \sim 140 \mathrm{~s})$ LMXRB GX $1+4$. The source, which appears to accrete from the wind of the $18 \mathrm{Mag}$, M5III companion, V2116 Ophiuchi (Davidsen et al. 1976), exhibited in the 1970s the highest spin up-trend ever observed in any pulsar with $\dot{v} / v$ : as high as $\sim 2 \%$ per year (Nagase 1989). The orbital period of the source was reported to be $\sim 304$ days by 
Pereira et al. (1999). More recently, Hinkle et al. (2003) reported a $P_{\text {orb }} \sim 1042$ days, based on infrared spectroscopic properties of V2116 Ophiuchi.

No observations were made from 1980 to 1982; later EXOSAT observations in 1983 and 1984 failed to detect the source, indicating an X-ray flux decrease of at least two orders of magnitude (Hall \& Davelaar 1983). In 1986, GX 1+4 was observed during a balloon flight (Greenhill et al. 1989) and exhibited a spin period of $111.8 \mathrm{~s}$, indicating that one or more spindown episodes occurred between 1980 and 1986. However, this episode must have been localized in time, since a collection of different data since 1980 shows that GX $1+4$ was again spinning up at an average rate of $6.6 \times 10^{-8} \mathrm{~s} / \mathrm{s}$ similar to the rate it had in the 1970s (Greenhill et al. 1989).

The pulsar reappeared with a reversed period derivative and low luminosity in 1987 (Makishima et al. 1988; Mony et al. 1991); and during 1989-1991, it continued to spin down rapidly with a mean rate about half, in absolute value, of the previous spin-up rate (Chakrabarty et al. 1997).

On an irregular basis, GX $1+4$ presents spin-up episodes with the absolute intensity similar to the spin-down trend. To explain such episodes on the basis of some apparent anticorrelation between torque and luminosity in the $20-60 \mathrm{keV}$ band seen by BATSE, several authors (Chakrabarty et al. 1997; Nelson et al. 1997) have suggested the formation of a retrograde disk implying a magnetic field of $B \sim 10^{12} \mathrm{G}$ on the surface of the neutron star. Instead, other authors (Beurle et al. 1984; Dotani et al. 1989; Mony et al. 1991) inferred the highest dipole magnetic field of any accreting pulsar from the torque reversal episodes: $B=(1-3) \times 10^{13} \mathrm{G}$ on the surface of the neutron star.

Cyclotron resonant scattering features (CRSFs) provide a tool for direct measurement of the magnetic-field strengths of accreting pulsars since they appear at $E_{\text {cyc }}=11.6 B_{12} \times(1+$ $z)^{-1} \mathrm{keV}$, where $B_{12}$ is the magnetic field strength in units of $10^{12} \mathrm{G}$ and $z$ is the gravitational red shift of the emitting region (Coburn et al. 2002; di Salvo et al. 2004). No CRSFs have been detected until today in the spectrum of GX $1+4$. This lack of detection is not surprising if the estimate based on the standard accretion torque theory is correct. In fact, the inferred magnetic field of $\sim 10^{13} \mathrm{G}$ converts to $E_{\mathrm{cyc}} \approx 100-300 \mathrm{keV}$, which is beyond the detection limit of hard X-ray satellites like Beppo-SAX and RXTE for the flux level and spectral shape of GX $1+4$ (Naik et al. 2005; Galloway 2000).

Moreover, evidence has been found that the hardness of the XRBP spectrum is closely related to the field intensity. Ginga, Beppo-SAX, and RXTE observations of binary accreting pulsars have revealed a correlation between line energy and spectral hardness in the high-energy part of the spectrum (Makishima \& Mihara 1992; dal Fiume et al. 2000; Coburn et al. 2002). Since the observed spectrum of GX $1+4$ is one of the hardest found among all X-ray binary pulsars, this relation suggests a magnetic field in excess of $10^{13} \mathrm{G}$. Similar results have been found for GS 1843-00 (Piraino et al. 2000).

In intermediate and high luminosity states $\left(L_{2-10 \mathrm{keV}}=\right.$ $10^{-10}-10^{-9} \mathrm{erg} \mathrm{cm}^{-2} \mathrm{~s}^{-1}$ ), its emission has been described either with a cut-off power law (Kotani et al. 1999; Cui 1997; Cui \& Smith 2004; Rea et al. 2005) or with a Comptonization model (Galloway 2000; Galloway et al. 2001; Naik et al. 2005). In both cases the source shows high intrinsic absorption and exhibits a strong iron line at $6.5-7 \mathrm{keV}$ with an equivalent width of $0.2-0.5 \mathrm{keV}$.

On an irregular basis, GX $1+4$ presents low luminosity states in which the flux decreases to a few $L_{2-10 \mathrm{keV}}=$ $10^{-11} \mathrm{erg} \mathrm{cm}^{-2} \mathrm{~s}^{-1}$. In these states, even though the other spectral characteristics are highly variable, the source always shows an enhanced $\mathrm{Fe}$ emission at $6.5-7 \mathrm{keV}$ (few $\mathrm{keV}$ equivalent width) and a harder spectrum in which the cut-off often cannot be found or is at higher energy with respect to the bright state (Rea et al. 2005; Naik et al. 2005; Cui 1997; Cui \& Smith 2004). These episodes can last from about one day (Galloway et al. 2000; Rea et al. 2005; Naik et al. 2005) to several months (Cui 1997; Cui $\&$ Smith 2004). The phenomenological interpretation is ambiguous: Cui \& Smith (2004) explain the low luminosity episodes as due to the propeller effect, while the residual power law emission is produced by accelerated particles; Galloway et al. (2000) present a model involving the accretion column disruption; Rea et al. (2005) suggest that the pulsar is hidden by a thicker accretion disk. In the last case, the residual radiation is produced by the reflection of the disk edge and by partial transparency of the medium at high energies $(\gtrsim 10 \mathrm{keV})$.

The main goal of this paper is to study the spectral and timing characteristics of GX 1+4 observed over a wide range of luminosity, within the INTEGRAL Galactic Plane Scan (GPS) part of the Core Programme (Winkler et al. 2003) from March 2003 until April 2004. The search for CRSF is also one of the key goals of our study.

In Sect. 2 we describe the observations and the data analysis method. In Sect. 3 we report on the results obtained from analysing light curves, spin period, pulse profiles and phaseresolved and phase-averaged spectra. In Sect. 4 we discuss our results in relation to previous observations. Finally, in Sect. 5 we draw our conclusions.

\section{Observations and data analysis}

The European Space Agency's International Gamma-Ray Astronomy Laboratory (INTEGRAL), launched in October 2002, carries 3 co-aligned coded mask telescopes.

- The imager IBIS (Imager on Board the INTEGRAL Satellite; Ubertini et al. 2003), which allows for 12 arcmin angularresolution imaging in the energy range from $15 \mathrm{keV}$ to $600 \mathrm{keV}$ with energy resolution of $6-7 \%$; IBIS is made of a low-energy (15-600 keV) CdTe detector ISGRI (Lebrun et al. 2003) and of a CsI layer, PICsIT (Labanti et al. 2003), designed for optimal performance at $511 \mathrm{keV}$, with an overall nominal energy range between $175 \mathrm{keV}$ and $10 \mathrm{MeV}$.

- The spectrometer SPI (Spectrometer on INTEGRAL; Vedrenne et al. 2003), sensitive from $20 \mathrm{keV}$ to $8 \mathrm{MeV}$ with an angular resolution of $2.5 \mathrm{deg}$ and an energy resolution of a few times $10^{-3}$.

- The X-ray monitor JEM-X (Joint European X-ray Monitor; Lund et al. 2003) made of two independent units JEM-XI and $J E M-X 2$, sensitive from $3 \mathrm{keV}$ to $34 \mathrm{keV}$, with an angular resolution of 1 arcmin and an energy resolution of $10-15 \%$.

As part of its core programme, INTEGRAL performs regular scans of the Galactic plane (GPS), with the aim of monitoring the timing and spectral properties of the known X-ray sources, discovering new transient sources, and mapping the diffuse emission of the Galactic plane (Winkler et al. 2003).

From March 21, 2003 to September 17, 2004 the region containing GX 1+4 was observed several times. In order to have the shadowgram of GX $1+4$ fully coded on the ISGRI detector plane, only pointings where GX $1+4$ is observed at less than 6 deg offaxis were used. This gives the best $S / N$ performance for ISGRI.

The data analysed in this paper consist of 435 science windows for a total observing time of $779 \mathrm{ks}$ and are divided into four intervals of semi-contiguous science windows 
Table 1. Summary of the INTEGRAL observations of GX 1+4. In the table we show the name for the observational period, its start and end time, the exposures of the three INTEGRAL instruments used here and the corresponding total number of source counts.

\begin{tabular}{lcccccccc}
\hline \hline Name & Start [MJD] & End [MJD] & \multicolumn{3}{c}{ Exposure [ks] } & \multicolumn{3}{c}{ Source counts } \\
& & & ISGRI & JEM-X & SPI & ISGRI & JEM-X & SPI \\
\hline OP1 & 52720.8076 & 52748.9027 & 72 & & & $2.9 \times 10^{5}$ & & \\
OP2 & 52917.6381 & 52921.7215 & 97 & & & $5.2 \times 10^{5}$ & & \\
OP3 & 53052.0923 & 53085.5729 & 155 & 47 & 137 & $1.9 \times 10^{6}$ & $2.0 \times 10^{5}$ & $4.2 \times 10^{3}$ \\
OP4 & 53252.4430 & 53287.3416 & 423 & 154 & 351 & $2.4 \times 10^{6}$ & $3.8 \times 10^{5}$ & $3.9 \times 10^{3}$ \\
OP3L & 53067.2531 & 53068.7772 & 32 & & & $9 \times 10^{4}$ & & \\
\hline
\end{tabular}

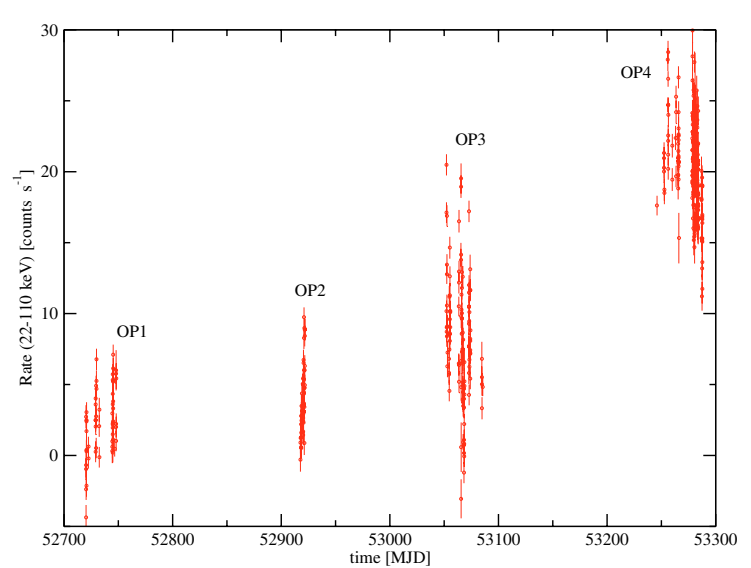

Fig. 1. Light curve of GX 1+4. We plot the INTEGRAL ISGRI count rates in the $22-110 \mathrm{keV}$ band during the galactic plane scan pointings with an off-set angle less than $6 \mathrm{deg}$. The binning is one science window corresponding to $1.6-3.6 \mathrm{ks}$. The time scale is modified Julian days $(\mathrm{MJD}=\mathrm{JD}-2400$ 000.5); MJD 52700 is 1 March 2003.

as shown in the light curve of Fig. 1. We define time intervals as follows: MJD 52720-52750 observational period 1 (OP1), MJD 52915-52925 (OP2), MJD 53050-53090 (OP3), and MJD 53 250-53290 (OP4). We also distinguish one sub-period corresponding to a low-flux episode in the middle of an intermediate flux state (OP3L), which started on MJD 53067.25 and ended on MJD 53068.75. In OP1, OP2, and OP3L, the source flux was too low for a detection with $J E M-X$ or SPI. In OP4 and in the last part of OP3 (after MJD 53072 ), it was possible to use only $J E M-X 1$, while in the first part of OP3 only $J E M-X 2$ was available. In OP3 we did not analyse the $14 \mathrm{ks}$ of $J E M-X 1$ data due to the low statistics; the reported exposure refers to $J E M-X 2$. The lower exposure time of $J E M-X$ compared to ISGRI is due to the smaller field of view of this instrument. The complete list of the observations is reported in Table 1.

We used version 5.1 of the INTEGRAL off-line standard analysis software (OSA 5.1), officially distributed by the INTEGRAL Science Data Centre (ISDC), to obtain the phase-averaged spectra of SPI and JEM-X. For the latter instrument we limited the analysis to the energy range $4-21 \mathrm{keV}$, for which the $J E M-X$ team ensures a reliable calibration as stated in the OSA handbook. All the information regarding the official software and the standard calibration can be found on the ISDC web-site at http://isdc . unige.ch/.

To analyse the ISGRI data we had to face two kinds of problems that are not optimally solved by the OSA pipeline: the lack of tools for performing straightforward timing and phaseresolved analysis and a not-yet-optimized energy reconstruction of the events. This required the development of specific tools, which are described in the Appendix.

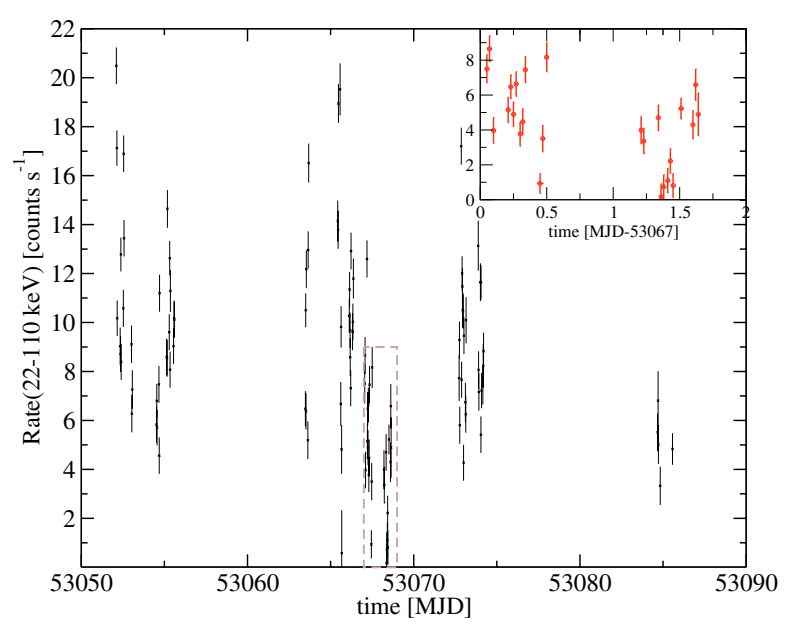

Fig. 2. Light curve of GX $1+4$ observed by ISGRI during the GPS for OP 3 in the $22-110 \mathrm{keV}$ energy band. The region with the dashed border highlights the low-luminosity episode, which is reproduced in the inset in the upper right corner. The binning is one science window corresponding to about $1.7-3.6 \mathrm{ks}$ depending on the operational mode. Errors are at $1 \sigma$ level.

\section{Results}

\subsection{Light curves}

The ISGRI light curve of GX $1+4$ in the $22-110 \mathrm{keV}$ energy range, averaged over the science window exposure (typically $1.6-3.6 \mathrm{ks})$, is shown in Fig. 1. During the INTEGRAL AO1 Core Program Monitoring, GX $1+4$ evolved from a weak state $\left(L_{20-40 \mathrm{keV}} \simeq 2 \times 10^{-10} \mathrm{erg} \mathrm{cm}^{-2} \mathrm{~s}^{-1}\right)$ to a much brighter one $\left(L_{20-40 \mathrm{keV}} \simeq 10 \times 10^{-10} \mathrm{erg} \mathrm{cm}^{-2} \mathrm{~s}^{-1}\right)$.

During OP3, from February 17, 2004 to March 23, 2004, the source exhibited erratic variations of about one order of magnitude in flux on a few kilo-seconds time scale (see Fig. 2). In particular, from MJD 53067.25 until MJD 53068.75, GX 1+4 underwent a very low luminosity state (OP3L) observed by INTEGRAL for $\sim 32 \mathrm{ks}$.

Since these low-luminosity episodes are peculiar in the behaviour of the source (Rea et al. 2005; Galloway 2000; Cui \& Smith 2004), OP3L data are analysed separately and excluded from the average analysis of OP3.

\subsection{Spin period}

Spin frequencies in the four observational periods were determined separately. First, we computed a preliminary power spectrum from the light curve binned with a $10 \mathrm{~s}$ time resolution. The $\sim 0.007 \mathrm{~Hz}$ peak position gave us a tentative period from which we produced a pulse profile $P_{\mathrm{t}}(\phi)$ for each science 

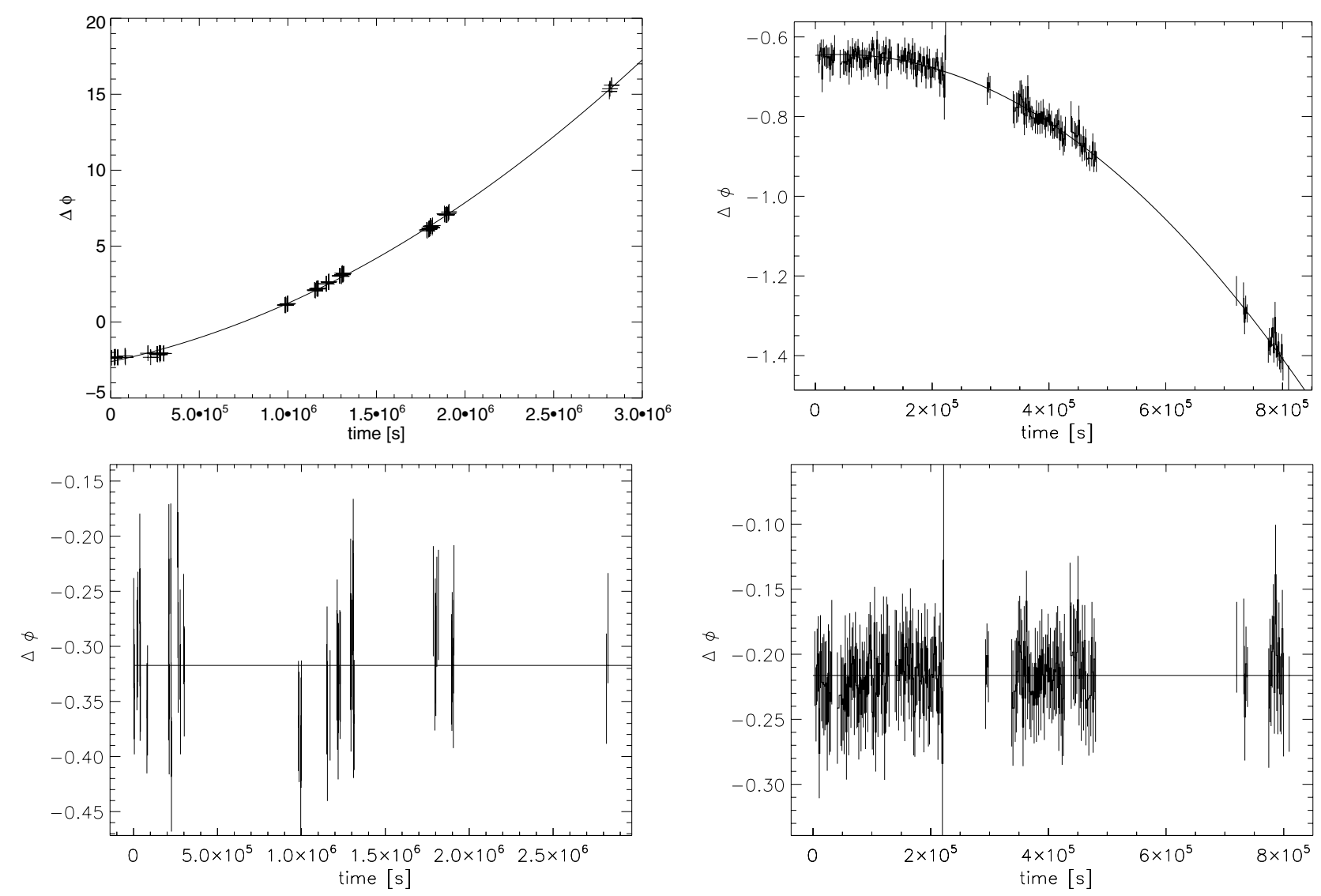

Fig. 3. The phase shifts are obtained using a constant folding period with the best-fit second-order polynomial overplotted (upper panels) and the residual phase shifts obtained with the folding parameters of Table 2 (lower panels). The phase shifts can be converted into time units by multiplying by the folding period of $140 \mathrm{~s}$. Left is for OP3 and right for OP4. The time scale is in seconds after the reference times of Table 2 .

window. Then, we computed the phase of the maximum of the pulse first Fourier component in each science window:

$\phi_{0}(t)=\tan ^{-1}\left(I_{\mathrm{c}}(t) / I_{\mathrm{s}}(t)\right)$,

where

$I_{\mathrm{c}}(t)=\int P_{t}(\phi) \cos (\phi) \mathrm{d} \phi \quad$ and

$I_{\mathrm{S}}(t)=\int P_{t}(\phi) \sin (\phi) \mathrm{d} \phi$

and $t$ is the science window central time ${ }^{1}$. If the law we used for folding is not optimal, $\phi_{0}(t)$ is not constant throughout the observational period; however, we can derive the optimal folding law from its variation. Let $\Delta \phi_{f}(t)=\phi_{0}(t)-\phi_{0}\left(t_{0}\right)$ be the phase shift measured after folding the data with the temptative period; we reconstruct the actual phase shift $\Delta \phi(t)$ by fitting $\Delta \phi(t)$ with a second-order polynomial

$\Delta \phi(t)=\phi_{0}+a\left(t-t_{0}\right)+b\left(t-t_{0}\right)^{2}$,

where $\phi_{0}$ is the starting phase shift determined by the fit and $t_{0}$ the reference folding time chosen close to the beginning of the observational period. The coefficient $a$ is used to correct the tentative choice of the folding frequency $v_{t}$ as

$v_{\mathrm{c}}=v_{t}-a$,

${ }^{1}$ For the pulse shape of GX $1+4$, most of the power is contained in the first harmonic; for sources with a double peak this method should be used with caution. where $v_{\mathrm{c}}$ is the corrected pulse frequency. The coefficient $b$ is linked to the pulse frequency derivative during the observational period by the relation

$\dot{v}_{\mathrm{c}}=-2 b$.

Finally, we verified that $\Delta \phi(t)$ obtained with the corrected folding law is constant within the errors and therefore the derived parameters are correct. A visual inspection of the residuals in Fig. 3 confirms that it is not necessary to use a higher-order polynomial and that the connection of phase shifts separated by wide gaps within the OPs is self consistent, even though in principle it could be non-unique. The different OPs cannot be phaseconnected due to their time separation and to the complex spin evolution of the source, with e.g. a torque reversal between OP3 and OP4.

Due to the low number of source counts for OP1, spin periods and their derivatives were only measured for OP2, OP3, and OP4. In Table 2 we summarise the values for the spin period and its first derivative, and the reference time at which they are measured. The relative time intervals are specified in Table 1. In some science windows in OP2 and OP3, the source was too weak for a good determination of the phase of the maximum, we so excluded them from the analysis, which produced an exposure time reduction of less than $5 \%$. The source shows the longest spin period of its observational history $P_{\mathrm{s}} \sim 141 \mathrm{~s}$, which confirms the overall spin down trend (Chakrabarty et al. 1997). In OP4 we find a sign inversion of the spin period derivative, which is the signature of a torque reversal episode. 


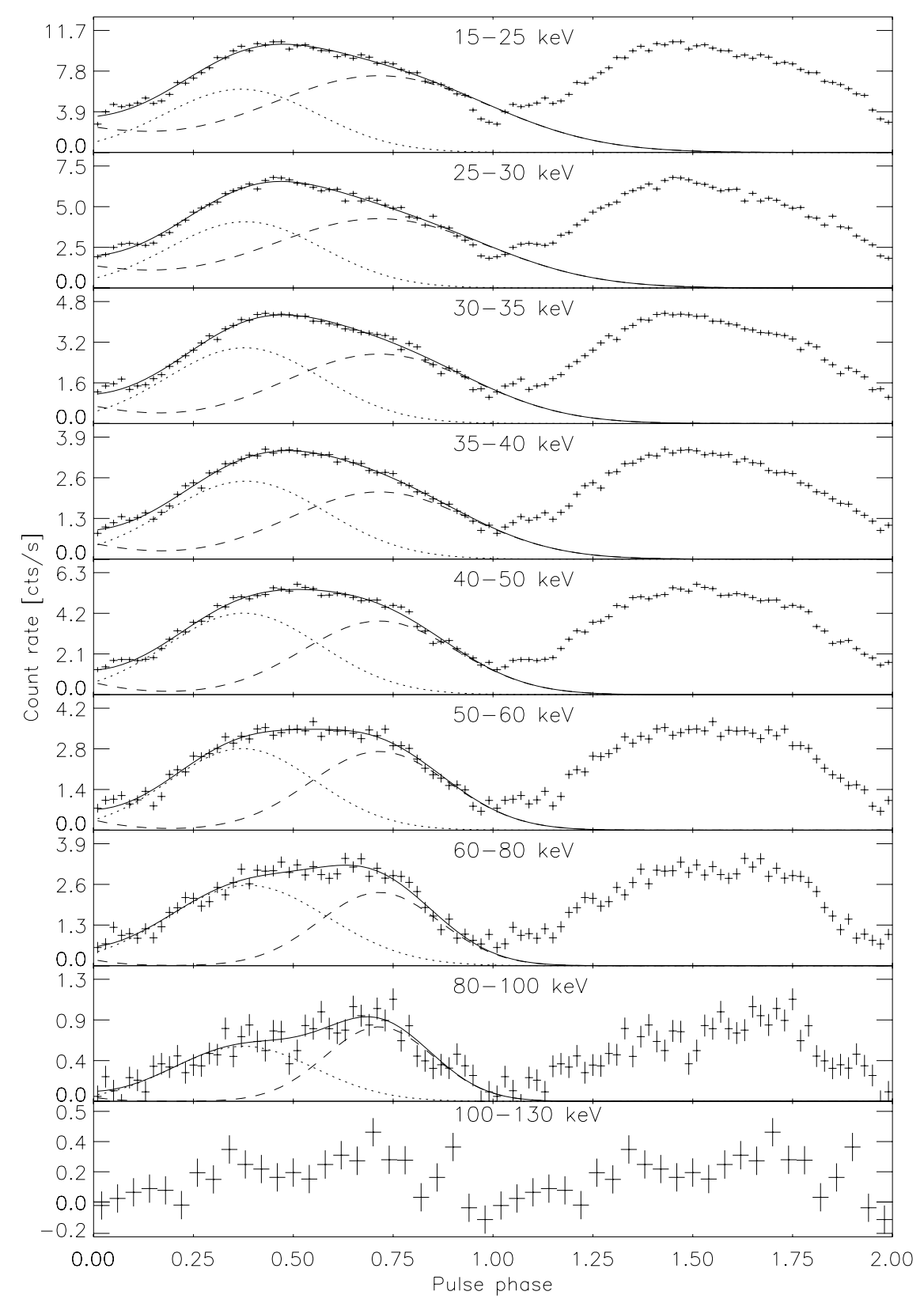

Fig. 4. The background subtracted pulse profiles of OP4 with energy ranges indicated in the figure. The vertical scale is in counts per second, the reference time, spin period, and spin-period derivative are listed in Table 2. The solid lines are the best-fit pulse profiles, the dotted and the dashed lines are the two Gaussian subcomponents used in the fit.

Table 2. Values for the spin period $(P)$ and spin period derivative $(\dot{P})$, in the three observational intervals where the source was bright enough for this determination, are reported. The errors are at $1 \sigma$ level computed from the fit to the phase shifts.

\begin{tabular}{llcc}
\hline \hline conv. name & reference time $[\mathrm{MJD}]$ & $P[\mathrm{~s}]$ & $\dot{P}[\mathrm{~s} / \mathrm{s}]$ \\
\hline OP2 & 52917.638038 & $139.630 \pm 0.006$ & $(1.5 \pm 0.1) \times 10^{-7}$ \\
OP3 & 53052.091969 & $140.6132 \pm 0.0002$ & $(1.034 \pm 0.002) \times 10^{-7}$ \\
OP4 & 53252.442934 & $141.56488 \pm 0.00014$ & $(-0.5331 \pm 0.0004) \times 10^{-7}$ \\
\hline
\end{tabular}

The secular spin-down trend we measured from a linear fit to the three measured pulse period values is $\dot{P}=(6.6 \pm 0.8) \times$ $10^{-8} \mathrm{~s} / \mathrm{s}$, but from the analysis of phase shifts we find local spin period derivatives that are larger in absolute value. This is consistent with the source behaviour in previous observations (Chakrabarty et al. 1997; Pereira et al. 1999): local spin-period variations are superimposed on a global spin down trend.

\subsection{Pulse profiles}

Using the values of $P$ and $\dot{P}$ in Table 2, we extracted the background subtracted pulse profiles of ISGRI for different energy intervals using the tools described in the Appendix B. The pulse profiles for OP4 are shown in Fig. 4, the ones for OP2, OP3, and OP4 in Fig. 5. Phase zero was arbitrarily chosen at the 
OP2

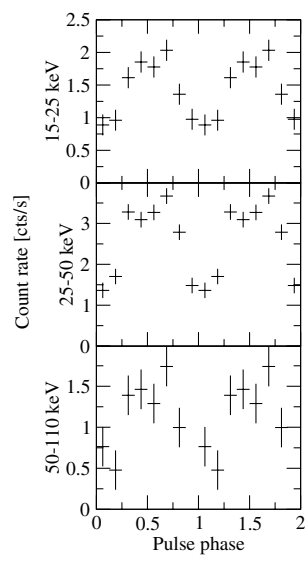

OP3
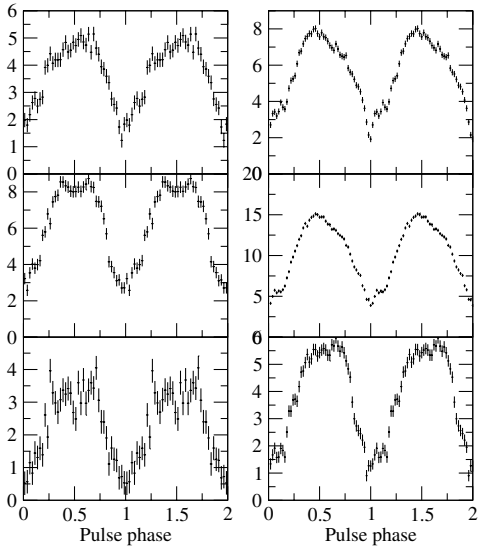

Fig. 5. The background subtracted pulse profiles for OP2, OP3, and OP4 in the energy ranges indicated in the plot. The reference time, spin period, and spin period derivative are listed in Table 2.

bottom of the dip in the $15-25 \mathrm{keV}$ profile for OP4 and at the pulse minimum for the other OPs. At the highest luminosity, the source is observed to pulsate up to $130 \mathrm{keV}$.

As a first approximation all pulse profiles show a single broad peak - as stated repeatedly in the literature. There are, however, clear substructures. A dip like structure within the broad minimum (around pulse phase zero) is evident at energies up to $60 \mathrm{keV}$ (Fig. 4). This is a well-known feature already found in RXTE and Beppo-SAX data (see e.g. Galloway et al. 2001; Naik et al. 2005). The pulse appears asymmetric and looks like a superposition of two (or more) peaks. This is also strongly suggested by various pulse profiles found in the literature (see e.g. Giles et al. 2000; Cui \& Smith 2004). Following this suggestion, we modelled our pulse profiles of OP4 by the sum of two Gaussian components (leaving out four data points that define the dip around phase 0). The model fits are shown as solid lines for all profiles below $100 \mathrm{keV}$ in Fig. 4. Each Gaussian is characterised by three parameters: centroid, width, and amplitude (a constant flux was set to zero since it was found to be unconstrained when it was left as a free parameter). Most fits are quite good (reduced chi-squared $\sim 1$ ), and others are acceptable (reduced chi-squared up to 1.4 for 40 d.o.f.). Our findings from these fits for OP4 (Fig. 4) are the following:

1. The centroids of both peaks are consistent with a constant in phase throughout the entire energy range: 0.39 and 0.71 , for the first and second peaks, respectively.

2. The widths of both peaks are slightly variable: while for energies above $40 \mathrm{keV}$ both peaks have a width consistent with 0.18 phase units, at lower energies the first peak becomes narrower while the second peak becomes wider (reaching 0.16 and 0.26 phase units, respectively, for $15-25 \mathrm{keV})$.

3. As evident from the visual inspection and from the ratio of the amplitudes of both peaks, the second peak is the dominating one. The ratio of amplitudes (first peak to second peak) increases from 0.5 at $15-20 \mathrm{keV}$ to 0.9 at $40-50 \mathrm{keV}$ with a subsequent small decrease to $\sim 0.7$ at $80-100 \mathrm{keV}$.

4. There is only a weak indication of a third small peak inside the pulse minimum around phase 0.1 (see, for instance, the 30-35 keV profile), reminiscent of the "bump" detected by Greenhill et al. (1989) and thereafter confirmed in other observations (e.g. Naik et al. 2005; Giles et al. 2000).

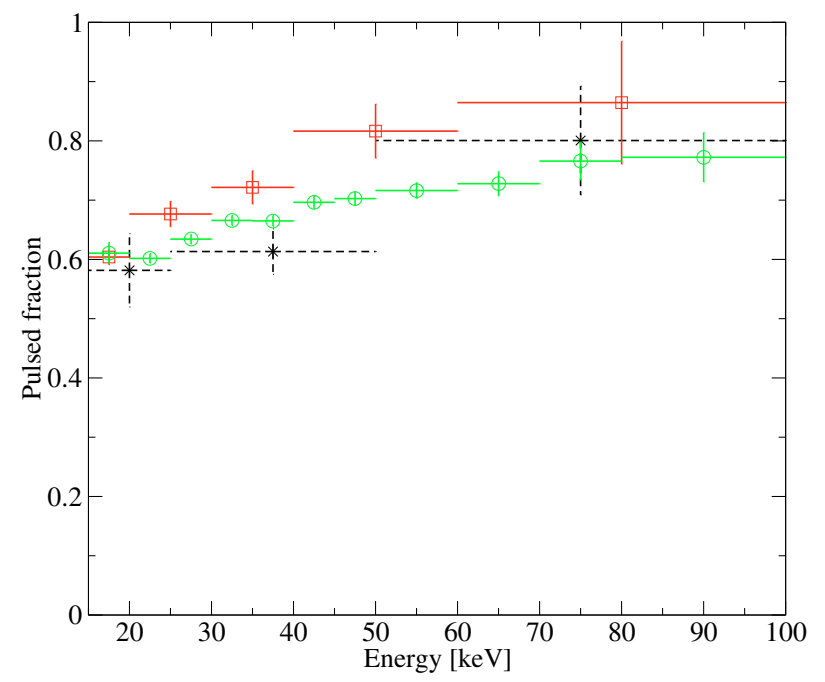

Fig. 6. The pulsed fraction in the three time intervals considered (see Table 2). The crosses refer to OP2 (weak), the squares to OP3 (intermediate), and the circles to OP4 (high luminosity); the errors are $1 \sigma$.

Pulse profiles of OP2 and OP3 (Fig. 5) confirm the general findings from OP4, although in OP2 and OP3 the two subpeaks contribute almost equally to the main pulse at all energies. An impressive demonstration of the existence of different emission components in GX 1+4 and their variability is given in Figs. 4 and 5 of Giles et al. (2000) where three peaks (and the dip structure) are very apparent.

To obtain the pulsed fraction we fitted the pulse profiles with a sine function, plus a constant and computed, from the sine amplitude $A$ and the constant $C$, the pulsed fraction as $2 A /(A+C)$. Pulsed fractions in different energy intervals (where the $S / N$ permitted such an analysis) are reported in Fig. 6. Interestingly, the intermediate luminosity observation (OP3) shows an average higher pulsed fraction in comparison with the brightest state (OP4). Pulsed fractions observed in OP2 are consistent with the others due to the large errors in the determination.

Using the spin period found for OP3, we extracted a pulse profile for the low-luminosity episode (OP3L) in the $15-100 \mathrm{keV}$ energy band. From the pulse profile of the right panel of Fig. 7 we can infer a pulsed fraction of $0.59 \pm 0.14$ using the method outlined above.

\subsection{Spectral analysis}

Spectral analysis was performed separately in the four OPs. Results for phase-averaged spectra are reported here while in Sect. 3.5 we describe our results on phase-resolved spectroscopy.

Broad-band spectral analysis, combining data from JEM-X, $I S G R I$, and SPI, was feasible only for OP3 and OP4, since the source was not detected by SPI and JEM-X in the lower luminosity states. Thus, we firstly analysed the ISGRI data alone for all luminosity states, and then we extended our analysis to the other instruments for the higher luminosity states.

The ISGRI spectra were fitted in the $17-110 \mathrm{keV}$ energy range using a cut-off power law $\left(N(E) \propto E^{-\Gamma} \exp \left[-E / E_{\mathrm{c}}\right]\right)$ with the best-fit parameters summarised in Table 3. In OP1 and OP2 the parameter determination is poor due to the weakness of the source, so the fit results can be considered as generally consistent with the others. Instead, we note a significant difference between the best-fit parameters of OP3 and OP4, which can be studied better by adding the data from JEM-X and SPI. 

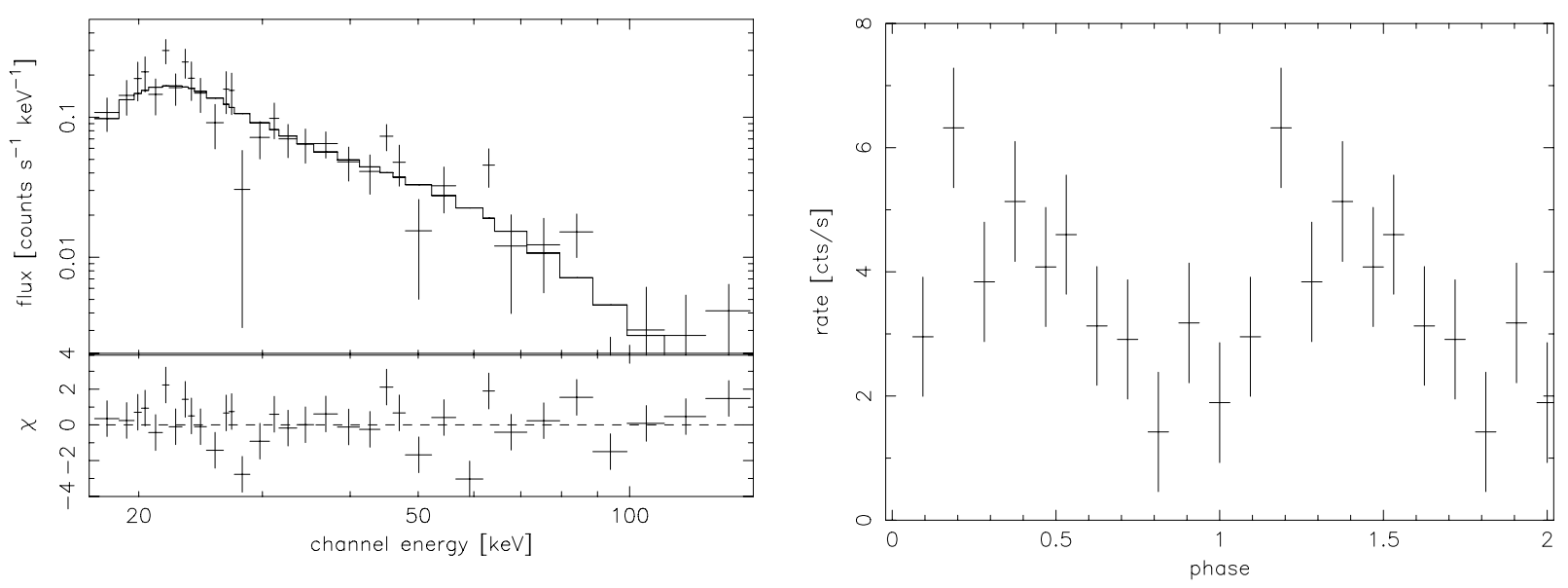

Fig. 7. The ISGRI spectrum (left) and the pulse profile (right) in the low-luminosity episode OP3L. The spectrum extends from 17 to $150 \mathrm{keV}$ with no break and is fitted by a power law with a slope of $2.5 \pm 0.2$. The pulse profile is obtained in the $15-100 \mathrm{keV}$ energy band.

Table 3. ISGRI spectral-fit parameters for the model $E^{-\Gamma} \exp \left(-E / E_{\mathrm{c}}\right)$ in the considered observing periods; OP3L is the low-luminosity episode. The flux is computed from the ISGRI data in the $20-40 \mathrm{keV}$ energy range and expressed in units of $10^{-10} \mathrm{erg} \mathrm{cm}^{-2} \mathrm{~s}^{-1}$. The uncertainties are at $90 \%$ c.l.

\begin{tabular}{lcccc}
\hline \hline & $\begin{array}{c}\text { Flux } \\
(20-40 \mathrm{keV})\end{array}$ & $\Gamma$ & $E_{\mathrm{c}}[\mathrm{keV}]$ & $\chi^{2} /$ d.o.f. \\
\hline OP1 & $1.7 \pm 0.5$ & $0.8 \pm 1.0$ & $22_{-9}^{+20}$ & $66 / 66$ \\
OP2 & $2.2 \pm 0.4$ & $0.9 \pm 0.6$ & $29_{-9}^{+16}$ & $55 / 66$ \\
OP3 & $4.9 \pm 0.3$ & $0.97 \pm 0.17$ & $27_{-3}^{+4}$ & $104 / 66$ \\
OP3L & $1.8 \pm 0.5$ & $2.5 \pm 0.2$ & $>150$ & $90 / 74$ \\
OP4 & $10.5 \pm 0.2$ & $0.34 \pm 0.05$ & $20.1 \pm 0.6$ & $97 / 66$ \\
\hline
\end{tabular}

The broad-band JEM-X, ISGRI, and SPI (4-110 keV) spectra for OP3 and OP4 were fitted first using a cut-off power law with low-energy absorption (WABS model in XSPEC) plus a Gaussian emission line at $\sim 6.5 \mathrm{keV}$ to model the blended neutral and ionized iron K-emission. The Gaussian emission line has parameters given by: $\left(N_{\mathrm{g}} / \sqrt{2 \pi \sigma_{\mathrm{g}}^{2}}\right) \exp \left(-\left(E-E_{\mathrm{g}}\right)^{2} / 4 \sigma_{\mathrm{g}}^{2}\right)$. Best-fit parameters are shown in Table 4 (left panel). The spectra were also successfully fitted with a Comptonization model (compTT in XSPEC) based on a spherical geometry using the analytical approximation of Titarchuk (1994) plus an additive Gaussian emission-line for the ionized K-emission from iron and lowenergy absorption. Best-fit parameters for this model are shown in Table 4 (right panel). The compTT model was used with approximation 2 and for red-shift $0 . k T_{0}$ is the temperature of the seed photons for the Comptonization, $k T_{\mathrm{e}}$ the temperature of the scattering electron cloud, while $\tau$ is its optical depth in spherical geometry. The cut-off model is slightly better for OP3, the compTT model may be preferred for OP4.

Significant differences between the spectra of OP3 and OP4 (at more than $90 \%$ c.l.) are found in the power law index and cut-off energy (cut-off model) and in the electron temperature and optical depth (compTT model). In Fig. 8 we plot the broadband count-rate and energy-flux unfolded spectra for OP3 and OP4 using the compTT model.

The visual inspection of the fit residuals (see Fig. 8 upper right panel) shows low points around $34 \mathrm{keV}$ that are reminiscent of a similar structure at a $2.5 \sigma$ level seen at the same energies in Beppo-SAX observations (Rea et al. 2005). However, the introduction of a multiplicative Gaussian absorption line
$N_{\text {abs }} \exp \left(-\left(E-E_{\text {abs }}\right)^{2} / 2 \sigma^{2}\right)$ does not improve the fit, since its significance is much less than $3 \sigma$.

The ISGRI spectrum of the low-luminosity episode (OP3L in Table 1) was also extracted, but JEM-X and SPI did not detect the source in this state. The spectrum (left panel of Fig. 7) was modelled by a power law with a slope of $2.5 \pm 0.2$ giving $\chi^{2} /$ d.o.f. $=90 / 74$. The PEXRAV model (Magdziarz \& Zdziarski 1995) proposed by Rea et al. (2005), which includes a reflection component besides the power law, is not constrained by our data.

\subsection{Phase resolved spectroscopy}

The OSA software (up to the current 5.1 version) does not provide tools for performing phase resolved spectroscopy for ISGRI and $S P I$. In the case of $J E M-X$ the task is very cumbersome. Thus we developed dedicated tools (described in the Appendix B) to analyse the ISGRI data.

Phase-resolved spectra of OP3 and OP4 were extracted according to the phase interval selection shown in the upper panel of Fig. 10 and modelled with a cut-off power law. In OP1 and OP2 the source was too weak for this kind of analysis. As far as OP3 is concerned, best-fit parameters do not change with the pulse phase within the statistical uncertainties. On the contrary, the best-fit parameters of phase-resolved spectra of OP4 (Table 5) change significantly with pulse phase: the power-law slope is not constant at a $99.9 \%$ confidence level, while the cut-off energy is not constant at a $90 \%$ confidence level.

Although not formally requested by the $\chi^{2}$ of the fit, we also searched for absorption-like features in the phase-resolved spectra. This was, again, motivated by the residuals observed in the spectrum of phase $0.6-0.85$ (Fig. 9), the descending part of the pulse, and by the weak evidence of such a line reported by Rea et al. (2005) and Naik et al. (2005). Using the Gaussian absorption model (see Sect. 3.4), the line centroid is found at $34 \pm 2 \mathrm{keV}$. The line width is not constrained by the fit and is set to $4 \mathrm{keV}$, compatible with the width of several absorption lines observed at similar energy in other sources (Coburn et al. 2002). The line significance using an F-test is $2.4 \sigma$, but, due to the questionable reliability of this statistical test in astronomical data analysis (Protassov et al. 2002), we conclude that the evidence for such a line, if any, is weak. However, systematic effects can be excluded because such a feature is not present in other pulse phases. 
Table 4. Results for the simultaneous fit of JEM-X, SPI and ISGRI spectra. The exposures are reported in Table 1 and the uncertainties of the bestfit parameters are at $90 \%$ confidence level. The physical units of the parameters are reported between squared brackets except for the unabsorbed flux, which is computed in the 2-60 keV energy range and expressed in units of $10^{-9} \mathrm{erg} \mathrm{cm}^{-2} \mathrm{~s}^{-1}$.

\begin{tabular}{lcclcc}
\hline \hline & Cut-off power law model & & & CompTT model & \\
parameter & OP3 & OP4 & parameter & OP3 & OP4 \\
\hline$N_{\mathrm{H}}\left[10^{22} \mathrm{~cm}^{-2}\right]$ & $10 \pm 3$ & $5.0 \pm 1.5$ & $N_{\mathrm{H}}\left[10^{22} \mathrm{~cm}^{-2}\right]$ & $<10$ & $25 \pm 2$ \\
$E_{\mathrm{g}}[\mathrm{keV}]$ & $6.8_{-0.6}^{+0.3}$ & $6.5 \pm 0.1$ & $E_{\mathrm{g}}[\mathrm{keV}]$ & $6.5 \pm 0.3$ & $6.6 \pm 0.3$ \\
$\sigma_{\mathrm{g}}[\mathrm{keV}]$ & $1_{-0.5}^{+1}$ & $<0.4$ & $\sigma_{\mathrm{g}}[\mathrm{keV}]$ & $0.5 \pm 0.5$ & 0.2 fixed \\
$N_{\mathrm{g}}\left[10^{-3} \mathrm{cts} \mathrm{cm}^{-2} \mathrm{~s}^{-1}\right]$ & $7_{-3}^{+6}$ & $2.7 \pm 0.5$ & $N_{\mathrm{g}}\left[10^{-3} \mathrm{cts} \mathrm{cm}^{-2} \mathrm{~s}^{-1}\right]$ & $4 \pm 2$ & $1.1 \pm 0.6$ \\
$E_{\mathrm{c}}[\mathrm{keV}]$ & $32 \pm 4$ & $21.8 \pm 0.7$ & $k T_{0}[\mathrm{keV}]$ & $1.7 \pm 0.3$ & $1.6 \pm 0.2$ \\
$\Gamma$ & $1.2 \pm 0.2$ & $0.51 \pm 0.05$ & $k T_{\mathrm{e}}[\mathrm{keV}]$ & $15.4 \pm 1.4$ & $13.1 \pm 0.2$ \\
& & & $\tau$ & $5.1 \pm 0.5$ & $6.80 \pm 0.15$ \\
Flux 2-60 keV & $1.77 \pm 0.07$ & $2.32 \pm 0.03$ & Flux 2-60 keV & $1.70_{-0.16}^{+0.04}$ & $2.16 \pm 0.04$ \\
$\chi^{2}$ d.o.f. & $212 / 212$ & $311 / 199$ & $\chi^{2} /$ d.o.f. & $224 / 211$ & $303 / 201$ \\
\hline
\end{tabular}
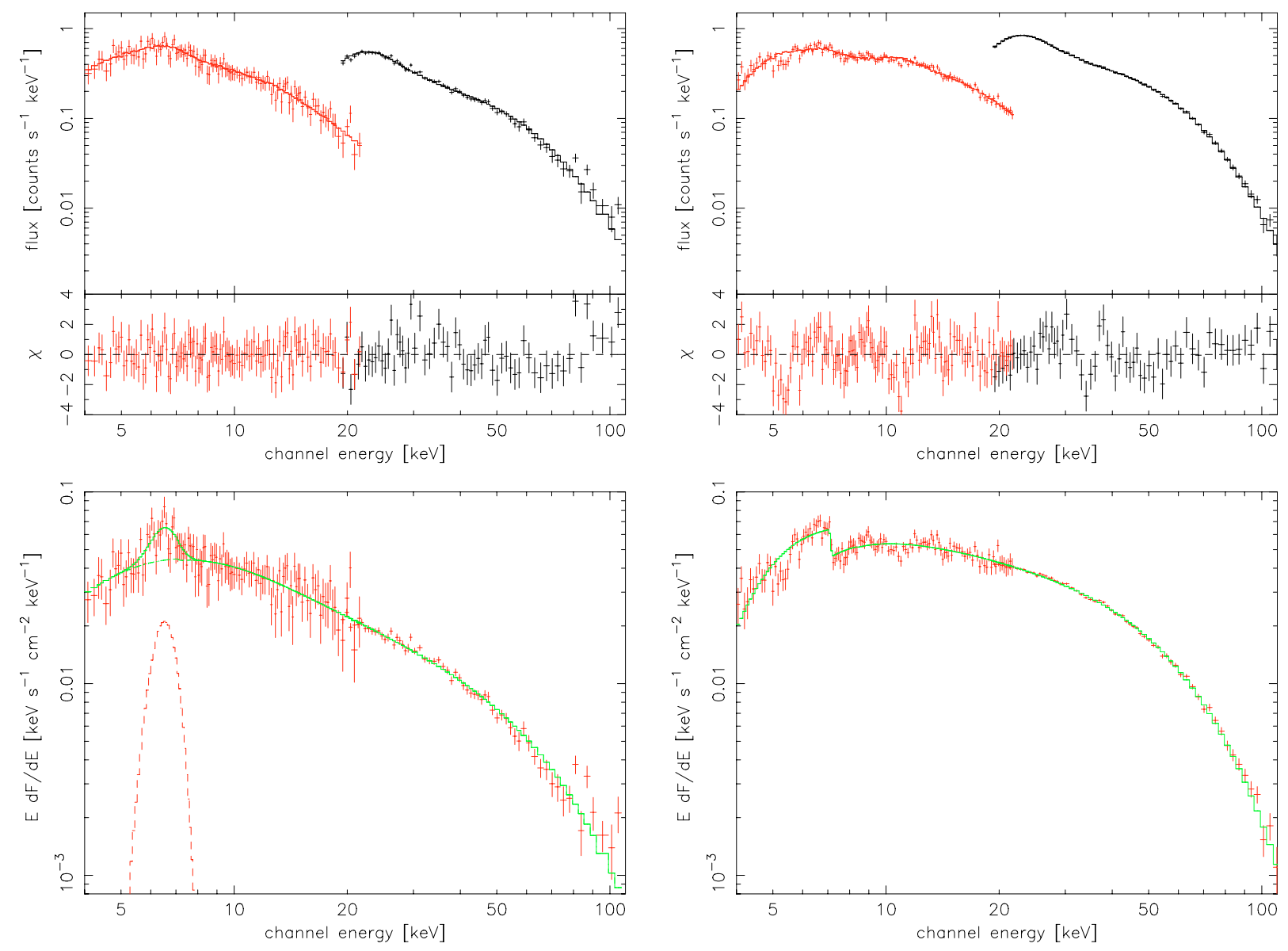

Fig. 8. Count-rate spectra (upper panels) and energy-flux unfolded spectra (lower panels) for OP3 (left) and OP4 (right) of GX 1+4. The model for the continuum is compTT plus a low-energy absorption and Gaussian emission line at $\sim 7 \mathrm{keV}$. The data between 4 and $21 \mathrm{keV}$ are from $J E M$-X, and the data extending from 19 to $100 \mathrm{keV}$ are from ISGRI.

\section{Discussion}

The main results of our analysis of the INTEGRAL observations of GX $1+4$ are:

1. The measurement of the longest spin period in the observational history of GX 1+4: $P_{\mathrm{s}} \sim 141 \mathrm{~s}$;

2. The measurement of the secular spin-down trend $\dot{P}=(6.6 \pm$ $0.8) \times 10^{-8} \mathrm{~s} / \mathrm{s}$, in agreement with previous findings;

3 . The detection of a variation from spin-down to spin-up associated with the high luminosity state;
4. Weak evidence in the phase-dependent spectra of an absorption feature with fundamental energy at $33-35 \mathrm{keV}$ that could be due to cyclotron resonant scattering;

5. The observation of a low-luminosity episode where the source is found pulsating and the spectrum does not show evidence of any reflection component.

In our INTEGRAL analysis we have also found, in line with previous observations from other satellites, evidence of energy dependent pulse profiles, spectral variations between luminosity states, and phase dependent spectra.

We confirm that the source is steadily spinning-down and maintaining a much lower luminosity than in the 1970s. The 


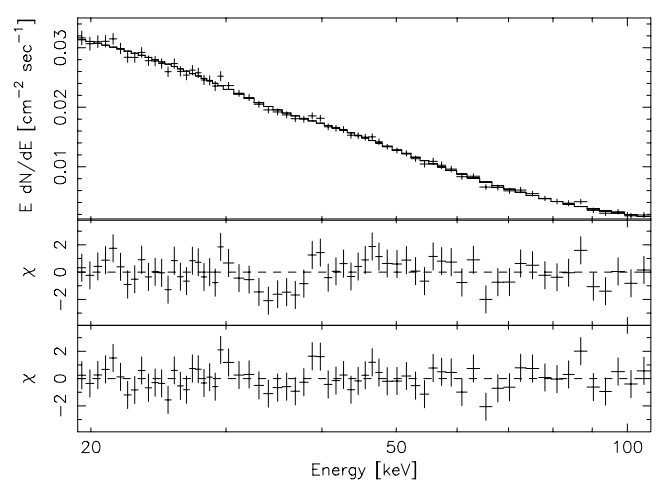

Fig. 9. In the upper panel we show the ISGRI energy-flux unfolded spectrum extracted in the phase interval $0.6-0.85$. The best-fit model has a multiplicative Gaussian absorption line at $(34 \pm 2) \mathrm{keV}$. The other panels show, from top to bottom, the residuals without the introduction of the line and the residuals with its introduction.

Table 5. Fit results for phase-resolved spectra, $\Delta \chi^{2}$ is referred to the passage from 72 to 70 d.o.f. with the introduction of a Gaussian absorption line. The $\sigma$ of the Gaussian absorption line is fixed at a value of $4 \mathrm{keV}$. The line significance is obtained using the F-test probability value. The uncertainties are the joint confidence limit at $90 \%$ probability.

\begin{tabular}{lcccc}
\hline \hline phase & $0.10-0.35$ & $0.35-0.6$ & $0.6-0.85$ & $0.85-1.10$ \\
\hline$\Gamma$ & $0.34 \pm 0.14$ & $0.10 \pm 0.08$ & $0.4 \pm 0.1$ & $0.6 \pm 0.2$ \\
$E_{\mathrm{c}}[\mathrm{keV}]$ & $19 \pm 1$ & $18.1 \pm 0.7$ & $22.5 \pm 1.5$ & $20 \pm 3$ \\
$E_{\text {abs }}[\mathrm{keV}]$ & & & $34 \pm 2$ & \\
$N_{\text {abs }}$ & & \multicolumn{3}{c}{$1.11 \pm 0.05$} \\
$\Delta \chi^{2}$ & & \multicolumn{3}{c}{$69(72) \rightarrow 56(70)$} \\
line significance & & \multicolumn{3}{c}{$2.4 \sigma$} \\
\hline
\end{tabular}

values of the measured spin-period derivative fall in the same range obtained on the basis of BATSE data. However, we observed a spin-up of the source in the high luminosity state: we exclude the possibility that the spin derivative inversion is due to a binary-system-induced Doppler shift, as already demonstrated by Pereira et al. (1999); it is more likely a signature of a torque-reversal episode, which is rather common in GX 1+4 (Chakrabarty et al. 1997). In our observation the torquereversal episode happened at a luminosity level compatible to the ones that Chakrabarty et al. (1997) reported as occurring at MJD 49300, 49700, and 50300. Unfortunately, on the basis of our INTEGRAL observation, it is not possible to narrow down the period at which the torque reversal occurred. It also needs to be stressed that a torque reversal does not imply such a luminosity value and vice versa as can be seen from Fig. 2 of Chakrabarty et al. (1997), where one sees spin-up episodes at lower luminosity and strong luminosity increases without a corresponding spin-up.

Considerations of the nature of the emission mechanism can be made by discussing the pulse-profile evolution with energy, together with the phase resolved spectroscopy. When the source is brighter $\left(L_{20-40 \mathrm{keV}}=10.5 \times 10^{-10} \mathrm{erg} \mathrm{cm}^{-2} \mathrm{~s}^{-1}\right.$, OP4 $)$, the pulse profile can be heuristically described by two Gaussian subpeaks centred at phases $\sim 0.4, \sim 0.7$ : the relative intensity between the first and the second peaks increases with energy (Fig. 4). At $\sim 20 \mathrm{keV}$ the pulse appears more peaked at an early phase while at energy just below $80 \mathrm{keV}$ the comparison with Fig. 5 shows that the two sub-peaks have equal intensity and the pulse shape becomes similar to the lower luminosity states observed in OP2 and OP3.

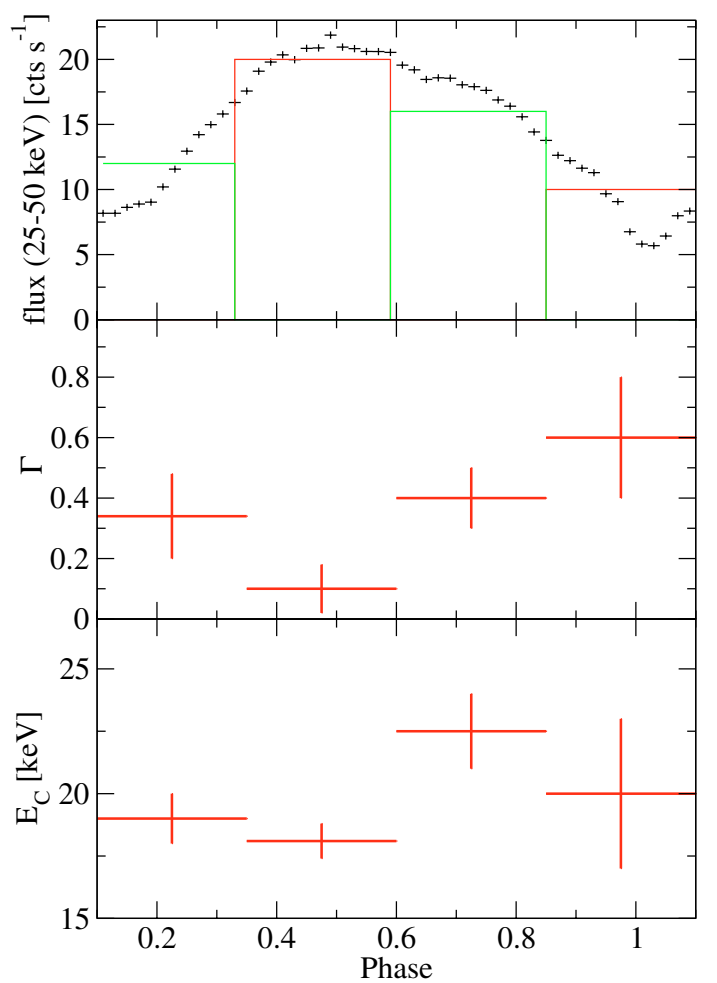

Fig. 10. The pulse profile in the $25-50 \mathrm{keV}$ energy band with the phase selection (upper panel), the slope (middle panel), and the cut-off energy (bottom panel) of the phase-resolved spectra of ISGRI in OP4. The best-fit parameters are obtained for the cut-off power-law model $E^{-\Gamma} \exp \left(E / E_{\mathrm{c}}\right)$ without the introduction of the Gaussian absorption line in the energy range $17-110 \mathrm{keV}$. The uncertainties represent the joint confidence limit at $90 \%$ probability $\left(\Delta \chi^{2}=2.7\right)$.

As the pulse shape changes with energy, the spectral properties evolve with phase: in the ascending and top part of the pulse the spectrum is well-fitted with a power law of slope $\sim 0.2$ and cut-off energy $\sim 19 \mathrm{keV}$, while in the descending part the spectrum softens. This suggests a larger optical depth for scattering in the first sub-peak with respect to the second sub-peak, which turns out to be more similar to the phase-averaged spectrum of OP3. Such a phase-dependent behaviour is different from the one observed in previous phase-resolved analyses (Galloway 2000; Galloway et al. 2001; Naik et al. 2005), which are focused on an absorption enhancement in correspondence with the pulse dip (at phase zero in our pulses) and do not find significant changes in the continuum within the remaining part of the pulse.

The broad-band JEM-X/ISGRI/SPI spectra are modelled either with an absorbed cut-off power law or with a Comptonization model. In OP1 and OP2, the fit parameters are not well-constrained. In OP4 (when the source is brighter), we find a harder emission up to $\sim 40 \mathrm{keV}$, followed by a faster decay, in comparison to OP3. In contrast to our results, Cui \& Smith (2004) find that when the source is bright $\left(\sim 10^{-9} \mathrm{erg} \mathrm{cm}^{-2} \mathrm{~s}^{-1}\right.$ in the $2-60 \mathrm{keV}$ energy band), the spectral index is very stable between 1 and 1.3 with $e$-folding energy at $28-40 \mathrm{keV}$. They also find that the fit parameters vary erratically with no apparent correlation to the flux on a time scale of a few days when the source is dimmer (spectral index between 0.2 and 2 and $e$-folding energy between 11 and $45 \mathrm{keV}$ ). These considerations imply that the spectral properties of GX $1+4$ are not determined uniquely 
by its luminosity state, but also depend on some still unknown parameter.

The picture is slightly clearer when we use a thermal Comptonization model: the value of the seed component temperature $\left(k T_{0} \simeq 1.6 \mathrm{keV}\right)$ is always similar to the one found with Beppo-SAX and RXTE, while the optical depth and plasma temperature are about the same but cannot be straightforwardly compared, confirming the variability of the accretion mechanism within a stable system. Limiting ourselves to our data, where the source is brighter, the optical depth is higher with respect to the lower luminosity state, while the temperature of the Comptonization plasma is lower. This spectral evolution with luminosity is consistent with the thermal Comptonization being the dominant emission process (see e.g. the conclusions in Becker \& Wolff 2005): using this model the larger accretion rate can explain the enhancements of both luminosity and optical thickness in the accreting plasma.

We observe weak evidence (at $2.4 \sigma$ level) of an absorption feature at $34 \pm 2 \mathrm{keV}$ in the descending part of the pulse profile, at phase [0.6-0.85] (see Fig. 9). Interpreting the absorption as due to the cyclotron resonant scattering of electrons, the magnetic field in the emitting region would be $(2.9 \pm 0.2) \times 10^{12}(1+z) \mathrm{G}$ where $z$ is the gravitational red shift of the emitting region. Its presence or enhancement just in the descending part of the pulse is typical of other systems such as 4U 0115+63 (Santangelo et al. 1999; Heindl et al. 1999) or Cen X-3 (Santangelo et al. 1998). The inferred magnetic field would be one order of magnitude less than expected from the standard accretion torque theory, but consistent with the one inferred from the model of emission due to a retrograde disk (Chakrabarty et al. 1997). Although the calibration uncertainties of ISGRI suggest that this weak evidence is an indication rather than a measurement, we note that a similar indication comes from PDS on board of Beppo-SAX. On the other hand, it is not surprising that $R X T E$ observations did not show spectral structures, although observations were carried out many times and in a variety of states (Giles et al. 2000; Cui \& Smith 2004). The single observations were shorter than those by INTEGRAL, and it is not possible to add many observations together because of the spectral variability of the source.

The low-luminosity episode observed with INTEGRAL is similar to the ones seen by Beppo-SAX (Rea et al. 2005; Naik et al. 2005) and RXTE (Galloway et al. 2000). The Beppo-SAX observation of such a low-luminosity episode showed a pulsed signal and a spectrum with an exponential roll-over. Cui \& Smith (2004) found with $R X T E$ that, when the pulsation disappears or is very weak, the spectrum can be modelled by a simple powerlaw with index $\sim 1.6$. They also find episodes of suppressed flux that show a pulsation and a detectable roll-over in the spectrum. During the low-luminosity episode observed by INTEGRAL, the source is found to pulsate, but a simple power law with a slope of $2.5 \pm 0.2$ models the spectrum of the source up to $150 \mathrm{keV}$ without the need of an exponential cut-off. This seems in contrast with Cui \& Smith (2004). On the other hand, we were not able to observe any signature of emission from a disk as found by Rea et al. (2005) or an enhancement of the absorption as found by Naik et al. (2005); this is due to the relatively high $(17 \mathrm{keV})$ lower threshold of our spectrum. In conclusion, with the analysed INTEGRAL observations we cannot settle the puzzle regarding the origin of these low-luminosity states.

\section{Conclusions}

In this paper we analysed the INTEGRAL data of the low-mass $\mathrm{X}$-ray pulsar GX $1+4$. The source was observed several times and found in different luminosity states with significant variations in its spectral and timing characteristics.

The overall spin-down trend is confirmed by the highest spin period ever observed for this source and by the negative local spin derivative we measured during most of the observation. However, in the high-luminosity state GX 1+4 showed a local spin-up. We estimate that the torque reversal happened at a source luminosity similar to analogous episodes described in Pereira et al. (1999). We notice that, during the spin up phase, the pulse profile is more peaked than in the intermediate luminosity state.

When the source was brighter, the spectrum showed a sharper exponential cut-off after becoming harder between 10 and $50 \mathrm{keV}$. This is consistent with the thermal Comptonization being the dominant emission process.

We also observed weak evidence of an absorption feature in the phase-resolved spectrum of GX $1+4$ at $33-35 \mathrm{keV}$ in the phase interval [0.6-0.85]. Rea et al. (2005) report a similar evidence in the phase-averaged spectrum obtained with Beppo-SAX. If this feature is due to cyclotron resonant scattering, the pulsar magnetic field would be $\sim 3 \times 10^{12} \mathrm{G}$.

Acknowledgements. The observational data used in this communication were collected by INTEGRAL, an ESA science mission for X-ray and Gamma-ray astronomy. The work was supported by the Italian Space Agency (ASI) under contract No. I/R/046/04 and ASI-INAF I/023/05/0, and by the German Space Agency (DLR) under contract Nos. 50 OG 9601 and 50 OG 0501. We thank Teresa Mineo and Nikolai von Krusenstiern for many useful comments on the manuscript. We also thank the anonymous referee for valuable comments and suggestions.

\section{References}

Barthelmy, S. D., Barbier, L. M., Cummings, J. R., \& SWIFT TEAM. 2005, Space Sci. Rev., in press [arXiv: astro-ph/0507410]

Becker, P. A., \& Wolff, M. T. 2005, ApJ, 630, 465

Beurle, K., Bewick, A., Harper, P. K. S., et al. 1984, Adv. Space Res., 3, 43 Bildsten, L., Chakrabarty, D., Chiu, J., et al. 1997, ApJS, 113, 367

Chakrabarty, D., Bildsten, L., Finger, M. H., et al. 1997, ApJ, 481, L101

Coburn, W., Heindl, W. A., Rothschild, R. E., et al. 2002, ApJ, 580, 394

Cui, W. 1997, ApJ, 482, L163

Cui, W., \& Smith, B. 2004, ApJ, 602, 320

dal Fiume, D., Orlandini, M., del Sordo, S., et al. 2000, in Broad Band X-ray Spectra of Cosmic Sources, Proceedings of the E1.1 Symposium of COSPAR Scientific Commission E, held during the 32nd COSPAR Scientific Assembly, 12-19 July, 1998 in Nagoya, Japan., ed. K. Makishima, L. Piro,

\& T. Takahashi (Pergamon Press for the Committee on Space Research), 399 Davidsen, A., Malina, R., \& Bowyer, S. 1976, PASP, 88, 606

Davidson, K., \& Ostriker, J. P. 1973, ApJ, 179, 585

di Salvo, T., Santangelo, A., \& Segreto, A. 2004, Nucl. Phys. B Proc. Suppl., 132,446

Dotani, T., Kii, T., Nagase, F., et al. 1989, PASJ, 41, 427

Galloway, D. K. 2000, ApJ, 543, L137

Galloway, D. K., Giles, A. B., Greenhill, J. G., \& Storey, M. C. 2000, MNRAS, 311,755

Galloway, D. K., Giles, A. B., Wu, K., \& Greenhill, J. G. 2001, MNRAS, 325, 419

Gehrels, N., Chincarini, G., Giommi, P., et al. 2004, ApJ, 611, 1005

Ghosh, P., \& Lamb, F. K. 1979a, ApJ, 232, 259

Ghosh, P., \& Lamb, F. K. 1979b, ApJ, 234, 296

Ghosh, P., Pethick, C. J., \& Lamb, F. K. 1977, ApJ, 217, 578

Giacconi, R., Gursky, H., Kellogg, E., Schreier, E., \& Tananbaum, H. 1971, ApJ, 167, L67

Giles, A. B., Galloway, D. K., Greenhill, J. G., Storey, M. C., \& Wilson, C. A. 2000, ApJ, 529, 447

Goldwurm, A., David, P., Foschini, L., et al. 2003, A\&A, 411, L223

Greenhill, J. G., Giles, A. B., Sharman, D. P., et al. 1989, A\&A, 208, L1

Gros, A., Goldwurm, A., Cadolle-Bel, M., et al. 2003, A\&A, 411, L179

Hall, R., \& Davelaar, J. 1983, IAU Circ., 3872, 1

Heindl, W. A., Coburn, W., Gruber, D. E., et al. 1999, ApJ, 521, L49 
Hinkle, K. H., Joyce, R. R., Fekel, F. C., Wood, P. R., \& Skrutskie, M. F. 2003, in ASP Conf. Ser., ed. R. L. M. Corradi, R. Mikolajewska, \& T. J. Mahoney, Astronomical Society of the Pacific, ASP Conf. Proc., 303, 129

Illarionov, A. F., \& Sunyaev, R. A. 1975, A\&A, 39, 185

Kotani, T., Dotani, T., Nagase, F., et al. 1999, ApJ, 510, 369

Labanti, C., Di Cocco, G., Ferro, G., et al. 2003, A\&A, 411, L149

Lebrun, F., Leray, J. P., Lavocat, P., et al. 2003, A\&A, 411, L141

Lund, N., Budtz-Jørgensen, C., Westergaard, N. J., et al. 2003, A\&A, 411, L231

Magdziarz, P., \& Zdziarski, A. A. 1995, MNRAS, 273, 837

Makishima, K., \& Mihara, T. 1992, in Frontiers Science Series, ed. Y. Tanaka, \& K. Koyama (Universal Academy Press), 23, Proceedings of the Yamada Conference XXVIII, held April 8-12, 1991, Aichi Sangyou Boueki Kaikan (Aichi Trade Center), Nagoya

Makishima, K., Ohashi, T., Sakao, T., et al. 1988, Nature, 333, 746

Mineo, T., Ferrigno, C., Foschini, L., et al. 2006, A\&A, 450, 617

Mony, B., Kendziorra, E., Maisack, M., et al. 1991, A\&A, 247, 405

Nagase, F. 1989, PASJ, 41, 1

Naik, S., Paul, B., \& Callanan, P. J. 2005, ApJ, 618, 866
Nelson, R. W., Bildsten, L., Chakrabarty, D., et al. 1997, ApJ, 488, L117

Pereira, M. G., Braga, J., \& Jablonski, F. 1999, ApJ, 526, L105

Piraino, S., Santangelo, A., Segreto, A., et al. 2000, A\&A, 357, 501

Pringle, J. E., \& Rees, M. J. 1972, A\&A, 21, 1

Protassov, R., van Dyk, D. A., Connors, A., Kashyap, V. L., \& Siemiginowska, A. 2002, ApJ, 571, 545

Rea, N., Stella, L., Israel, G. L., et al. 2005, MNRAS, 364, 1229

Santangelo, A., del Sordo, S., Segreto, A., et al. 1998, A\&A, 340, L55

Santangelo, A., Segreto, A., Giarrusso, S., et al. 1999, ApJ, 523, L85

Segreto, A., \& Ferrigno, C. 2006, in Proceedings of the 6th INTEGRAL

Workshop The Obscured Universe, in press

Stella, L., White, N. E., \& Rosner, R. 1986, ApJ, 308, 669

Titarchuk, L. 1994, ApJ, 434, 570

Toor, A., \& Seward, F. D. 1974, AJ, 79, 995

Ubertini, P., Lebrun, F., Di Cocco, G., et al. 2003, A\&A, 411, L131

Vedrenne, G., Roques, J.-P., Schönfelder, V., et al. 2003, A\&A, 411, L63

Winkler, C., Gehrels, N., Schönfelder, V., et al. 2003, A\&A, 411, L349 
C. Ferrigno et al.: INTEGRAL observation of GX $1+4$, Online Material $p$ I

\section{Online Material}




\section{Appendix A: ISGRI energy calibration issues}

The ISGRI detection layer consists of a $128 \times 128$ array of independent CdTe detector pixels (Lebrun et al. 2003). This kind of detectors is affected by the "Charge Loss Effect": since in CdTe the hole lifetime is much shorter than its transit time, the induced current can be significantly reduced depending on the position of the interaction on the detector. To reconstruct the energy of the incoming photons, it is necessary to perform a correction of the pulse amplitude value as a function of the pulse rise time; for this purpose OSA software makes use of multiplicative coefficients stored in a "Look-Up" Table (LUT2).

The LUT2 distributed with the OSA software is not optimized, especially in an energy region near $80 \mathrm{keV}$ where a fraction of the photons is overcorrected. Ad hoc absorption-like features at $\simeq 80 \mathrm{keV}$ have been introduced in the OSA 4.2 and OSA 5.1 ISGRI Effective Area (respectively dashed and solid lines in Fig. A.1) modelled on the basis of the observed Crab spectrum assumed to be a power law with index 2.2. This choice of the index does not agree with the widely accepted value of 2.1 (Toor \& Seward 1974). ISGRI spectra are thus systematically softer than other instruments. Moreover, the introduction of these ad hoc corrections in the effective area could produce the appearance of fake features in the spectra of sources that are different from the Crab.

To improve the calibration, a new LUT2 based on on-ground and in-flight calibration data has been generated. Using this new LUT2 a better charge loss correction is obtained ${ }^{2}$.

We also generated new spectral-response matrices taking into account the charge loss effect and its correction by the new LUT2. The effective area, shown as the dash-dotted line in Fig. A.1, does not exhibit any $80 \mathrm{keV}$ absorption-like feature. This constitutes a confirmation of the better energy correction performed with the new LUT2: there is, in fact, no physical reason for this feature.

Also the spectral parameters we obtained with our response matrices are closer to the literature values (e.g. an index 2.1 for the Crab), while the $\chi^{2}$ values of the fits are systematically better than the ones obtained using the OSA 4.2 and 5.1 calibration files. In Fig. A. 2 the fit to the Crab spectrum can be appreciated, but the reader can read a more detailed discussion in the appendix of Mineo et al. (2006).

If one assumes that the fractional spread of the Crab residuals of Fig. A. 2 is an empirical indicator of the calibration systematic errors, the calibration systematics can be neglected for our observation in OP4. In fact, the Crab reference spectrum we used and the source spectrum have about the same number of total counts in the $20-40 \mathrm{keV}$ energy range but the $S / N$ of the Crab is three times higher.

\section{Appendix B: Timing analysis of the ISGRI data}

The standard OSA pipeline does not allow to perform timing analysis of pulsars. We then developed an alternative software ${ }^{3}$ to extract simultaneously spectra, light curves and phase resolved spectra from the event lists; a dedicated paper which

\footnotetext{
${ }^{2}$ For the technical details we refer to Alberto Segreto's talk at the Internal INTEGRAL workshop available at

http://www.rssd.esa.int/Integral/workshops/Jan2005/ and to the new calibration files available at

http://www.ifc.inaf.it/ ferrigno/integral/

3 The software can be downloaded at the URL

http://www.ifc. inaf.it/ ferrigno/INTEGRALsoftware.html
}

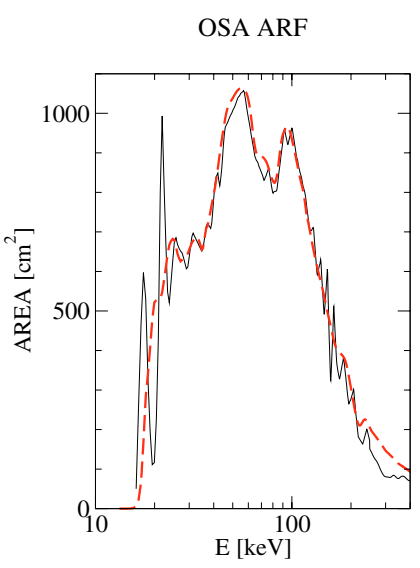

ADOPTED ARF

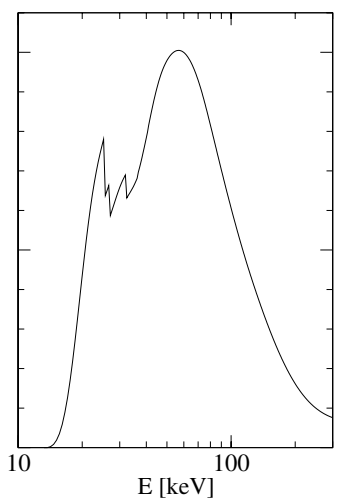

Fig. A.1. Comparison between the effective area as distributed with OSA (left panel) and the new effective area obtained after performing the energy correction with the new LUT2 (right panel). In the left panel the dashed line is the effective area distributed with OSA 4.2, and the solid line the effective area distributed with OSA 5.1. The OSA effective areas present many bumps and wiggles, which are introduced ad hoc to compensate for the OSA LUT2 energy correction. Features in the $30-40 \mathrm{keV}$ region are due to CdTe absorption edges.

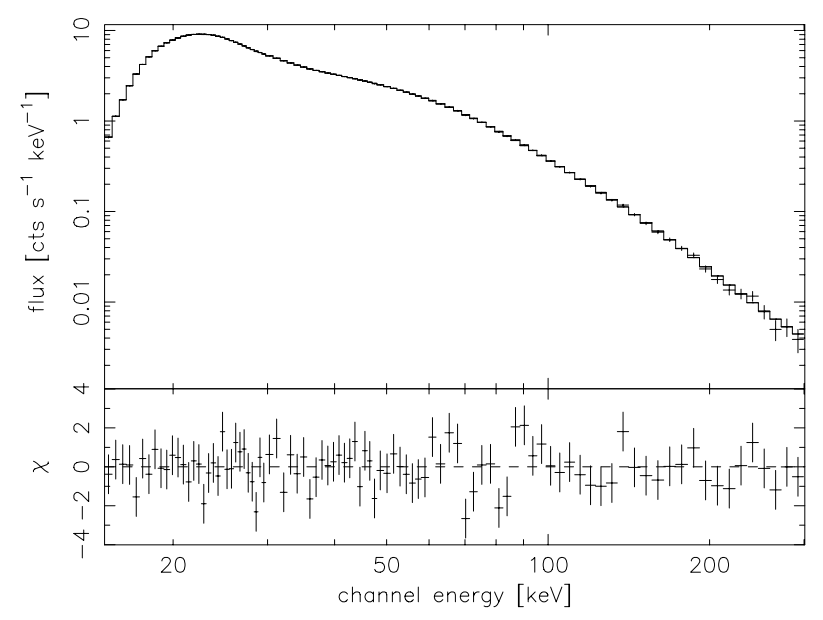

Fig. A.2. Crab spectrum from a selection of science windows in the revolutions 102 and 103 in staring observations, the exposure is about $47 \mathrm{ks}$. The fit is obtained with a power law (slope 2.1) without adding systematic errors.

describes the method in detail is in preparation, here we give a brief overview.

When illuminated by an X-ray source, a coded mask casts a shadow on the detector plane (shadowgram) that, correlated with the known mask pattern, allows to obtain the position and intensity of the sources within the instrument field of view (Goldwurm et al. 2003).

To obtain spectra and light curves of these sources, a shadowgram deconvolution process can be repeated several times, each time after selecting events in the energy and time intervals of interest. However, when many narrow energy or time bins are considered, a large amount of CPU time is required.

A much faster way to obtain light curves or spectra from coded mask telescopes when the source positions have already been determined (because known a priori or predetermined by a shadowgram deconvolution), is via the use of the illumination pattern that the mask casts on the detection plane. Given the pixellated nature of the detector plane, some of the detector pixels will then be fully illuminated by a source while other 


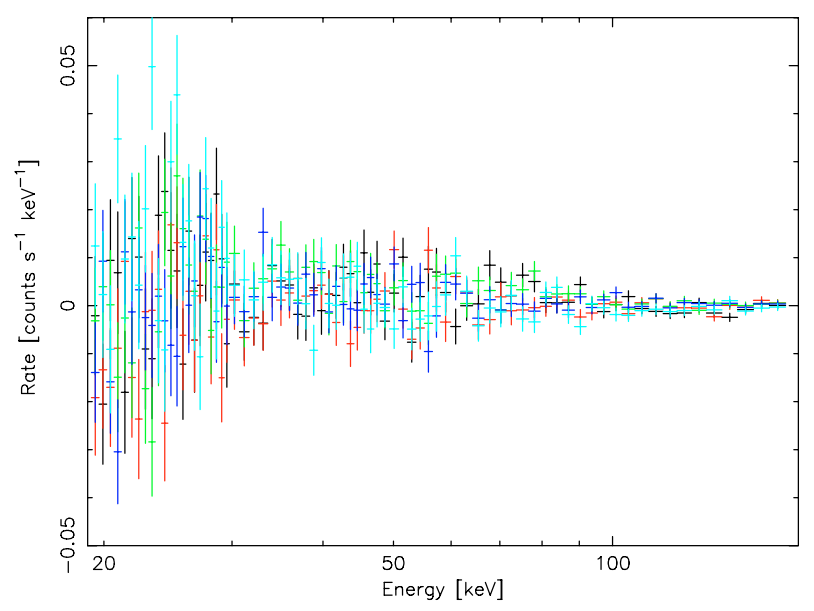

Fig. B.1. Spectra extracted in five positions at two degrees from GX 1+4 for the OP4 data set.

pixels will be partially illuminated or not illuminated at all. For each pixel the amount of illumination from each source, normalized to the maximum illumination value is called the pixel Photon Illumination Fraction (PIF).

Using this PIF information, spectra, light curves, or other scientific products can be extracted by means of a minimum amount of algebraic operations, without the need of repeating a shadowgram deconvolution process over and over again. This method, which is used e.g. by the standard pipeline of BAT (Barthelmy et al. 2005) on board Swift (Gehrels et al. 2004) and by the $J E M-X$ software, does not account for the presence of multiple sources in the same field of view. Due to a non ideal imaging point-spread function this may produce cross contamination between the signals from different sources. To avoid this problem, we then developed a decontamination algorithm, based again on simple algebraic operations (Segreto \& Ferrigno 2006).

To validate our extraction method we compared the background-subtracted spectra of several sources with the one obtained using the OSA standard pipeline and verified that the two methods give equivalent results not only in terms of spectral shape but also in their $S / N$.

The PIF-based extraction method we developed is not only much faster, but it also allows to easily perform fast timing analysis and phase-resolved spectroscopy, tasks not supported by the standard OSA software.

To estimate the reliability of the background rejection we extracted spectra in several sky directions chosen around the main source to ensure the same observational conditions but far enough to avoid problems due to the $12^{\prime}$ point-spread function (Gros et al. 2003). In Fig. B.1 we plot an example for five pointings within OP4. As shown, the spectra are all consistent with zero without points with $S / N$ exceeding $\sim 3$ in each spectrum. We verified that the $S / N$ distribution of the spectra in the $19-110 \mathrm{keV}$ range is described well by a Gaussian function with $\sigma \sim 1$. 